\title{
Transatlantica
}

Revue d'études américaines. American Studies Journal

$1 \mid 2012$

Le roman policier, littérature transatlantique / Maisons Hantées

\section{Richard Pak : Pursuit}

Du mercredi 24 octobre au dimanche 6 janvier 2013 Galerie du Château d'Eau (Toulouse)

\section{Muriel Adrien}

\section{(2) OpenEdition}

\section{Journals}

Édition électronique

URL : https://journals.openedition.org/transatlantica/5852

DOI : 10.4000/transatlantica.5852

ISSN : 1765-2766

Éditeur

Association française d'Etudes Américaines (AFEA)

Référence électronique

Muriel Adrien, « Richard Pak: Pursuit », Transatlantica [En ligne], 1 | 2012, mis en ligne le 12 décembre 2012, consulté le 14 février 2023. URL : http://journals.openedition.org/transatlantica/5852 ; DOI : https://doi.org/10.4000/transatlantica.5852

Ce document a été généré automatiquement le 14 février 2023.

\section{(c) (i) $\odot$}

Creative Commons - Attribution - Pas d'Utilisation Commerciale - Pas de Modification 4.0 International - CC BY-NC-ND 4.0

https://creativecommons.org/licenses/by-nc-nd/4.0/ 


\section{Richard Pak : Pursuit}

Du mercredi 24 octobre au dimanche 6 janvier 2013 Galerie du Château d'Eau (Toulouse)

\section{Muriel Adrien}

1 A la première page du catalogue de l'exposition Pursuit, figure le portrait d'une jeune fille qui tire son débardeur élimé vers le bas pour révéler à hauteur du cœur un tatouage en négatif qui ne peut se lire que de droite à gauche (et avec difficulté) dans la page-miroir argentée en vis-à-vis à gauche: "Follow your Dreams." En lisant cet aphorisme, le lecteur-spectateur aperçoit aussi inévitablement son lancinant reflet déformé en arrière-plan. En dernière page, pour boucler la boucle, des lettres géantes placardées 'H-O-P-E' se découpent sur l'horizon d'un ciel laiteux, en haut d'une colline déserte ou d'un faux plat, tel un mirage. ${ }^{1}$ En vis-à-vis encore, mais à droite (puisque HOPE est à lire de gauche à droite, cette fois-ci), se trouve l'indéfectible miroir de l'autre côté duquel on ne passera pas, car ainsi s'achève l'ouvrage. 

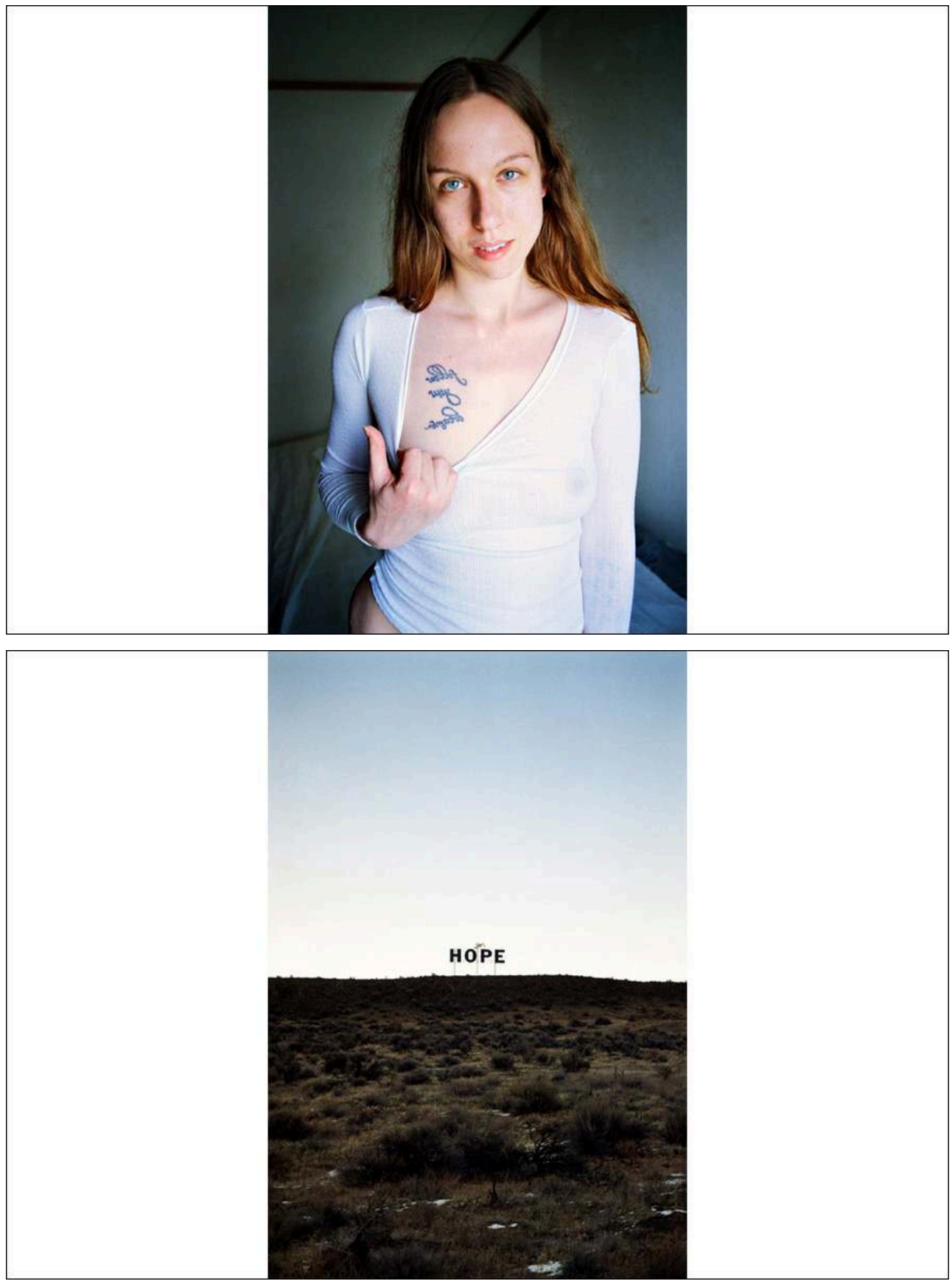

2 Le ton est donné. C'est l'image inversée d'un American dream que nous donne à voir Richard Pak. Il recueille les rêves avortés ou désenchantés des laissés-pour-compte, ou les rêves sans âme de la middle-class de la banlieue pavillonnaire uniformément rangée. Ne nous y trompons pas: notre reflet brouillé dans la page-miroir n'indique pas une quelconque prétention à l'universalité dans ces clichés à la couleur locale passée. Tout au plus peut-on y voir la projection d'une certaine subjectivité, celle-là même de l'auteur qui nous livrerait un imaginaire nourri aux livres et films américains. 

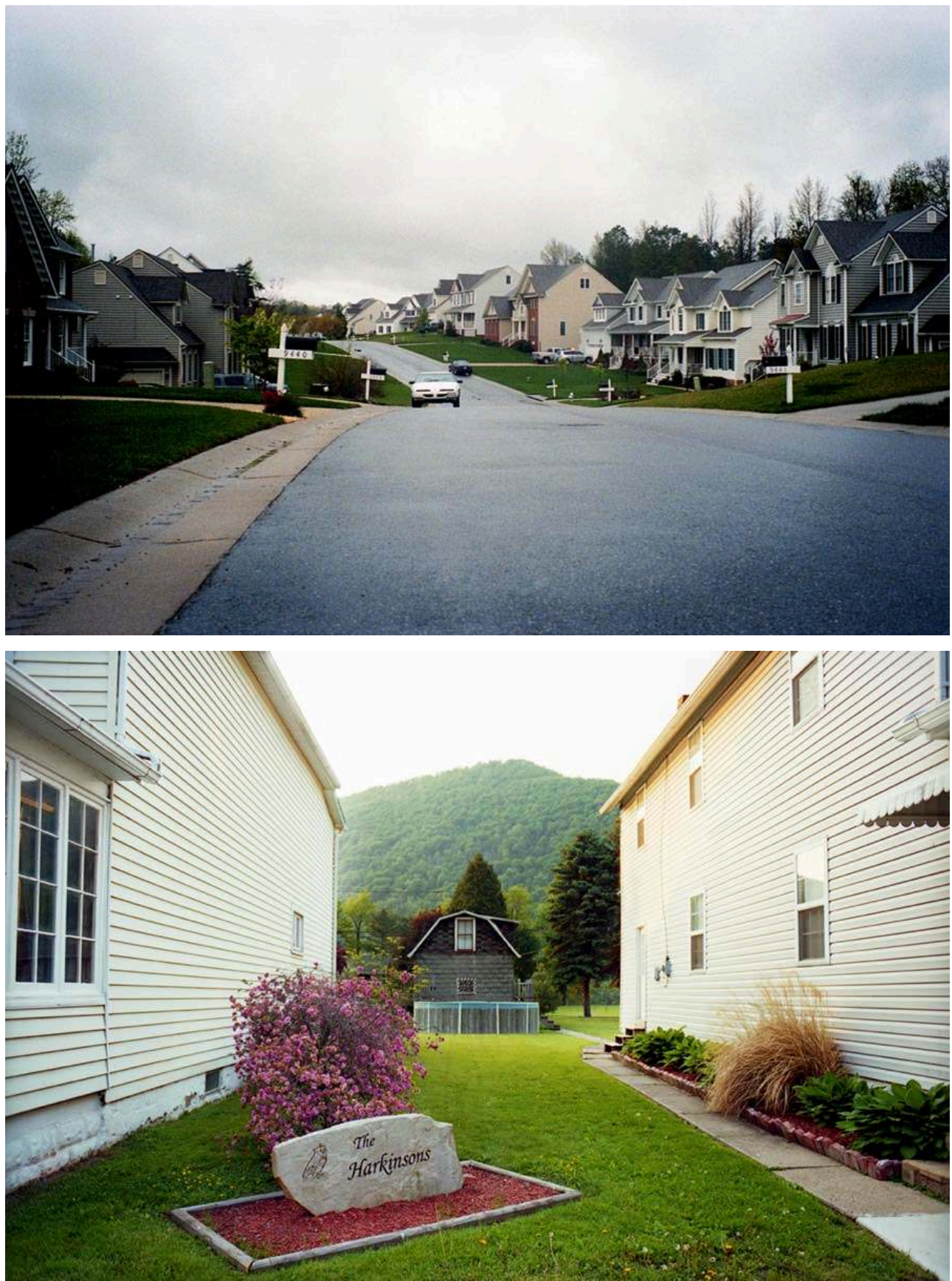


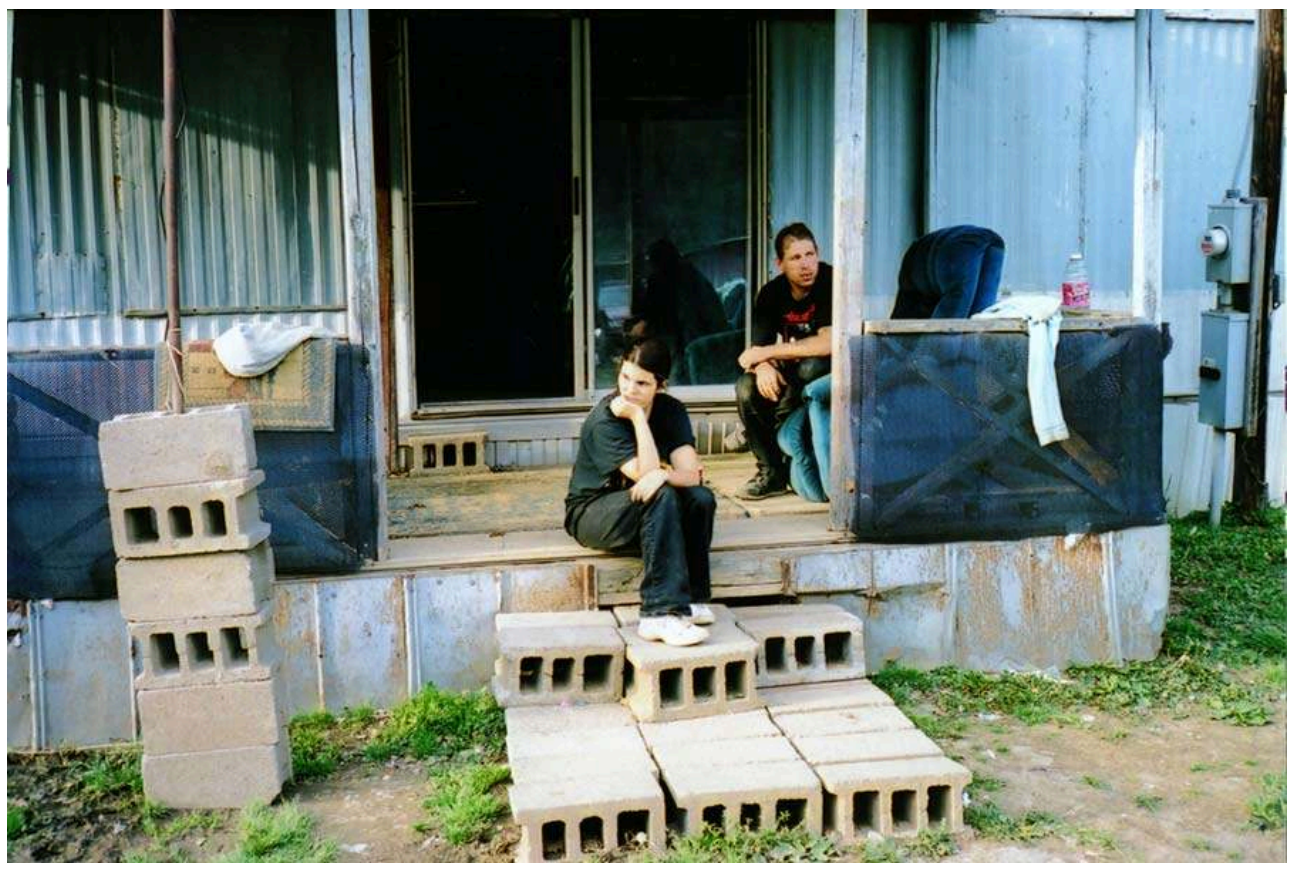

3 Le titre de l'exposition, quant à lui, Pursuit, renvoie à l'un des principes intangibles et inaliénables de la Constitution américaine, "the pursuit of happiness" - principe quasi poétique, en tout cas chimérique, que Richard Pak trouve insolite dans un texte institutionnel ${ }^{2}$. Cette quête du bonheur est ce à quoi les personnes photographiées devraient pouvoir prétendre, ce bonheur fragile et incertain des oubliés de la prospérité américaine; mais il renvoie aussi à la quête du photographe qui poursuit également ses propres rêves.

4 Au cœur de l'étage inférieur du Château d'eau, en fin de parcours, des pans de murs sont occupés par des extraits textuels du journal de bord dans lequel Richard Pak a consigné au quotidien ses aventures américaines, les souvenirs de ses rencontres. La lecture devient vite captivante malgré l'inconfort de la station debout prolongée. En témoigne la grande densité de visiteurs. C'est que l'auteur nous y livre les clefs de sa démarche. Et l'on comprend enfin les intuitions glanées çà et là devant les oeuvres. Son journal de bord dit d'abord le caractère narré de son propos, la linéarité temporelle de sa trajectoire on the road, de son beat trip, de ses aventures comme autant de petites nouvelles ou short cuts dans un contexte bien défini et repérable. Il reproduit dans son écriture-même un déroulement narratif comme en écho aux références qu'il se donne dans sa quête d'images, références littéraires et cinématographiques. Foisonnent des allusions à Carver, Kerouac, Russell Banks (Trailer Park), Truman Capote, John Fante ${ }^{3}$ ou au cinéma New Hollywood des années $1970{ }^{4}$ 


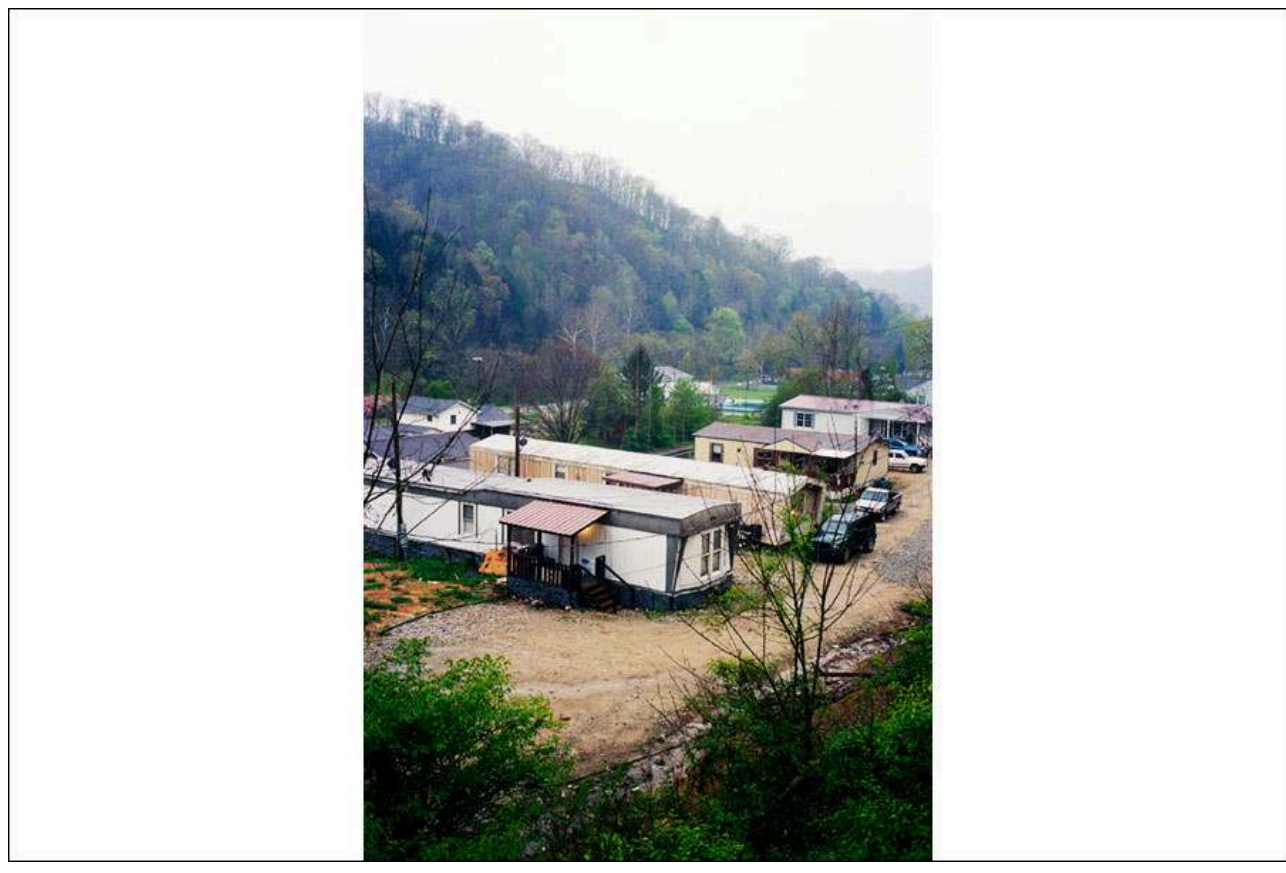

5 Ces citations sont plus que des résonances ou des clins d'œil, ce sont les visées de son objectif. Richard Pak dit ne pouvoir envisager le travail sans son texte. Dans la revue Photos nouvelles, il écrit: "En jouant sur les variations des thèmes de l'ennui, de l'isolement, voire de l'abandon (d'un proche, d'un idéal ?), j'essaie de faire entendre ce que résume Pierre-Yves Pétillon (in Histoire de la littérature américaine), cette voix si américaine faite de solitude et de stoïcisme, où l'on perçoit, comme dans un tableau d'Edward Hopper, un mélange de mélancolie léthargique et de nostalgie des lointains" $\left(\mathrm{n}^{\circ} 51,18\right)$. Richard Pak dit s'incrire dans un genre initié par Les Américains de Robert Frank, mais son projet photographique est avant tout un retour aux sources: Please, come again est le titre de son catalogue. Il s'agit d'aller photographier les représentations qu'il reconnaît.

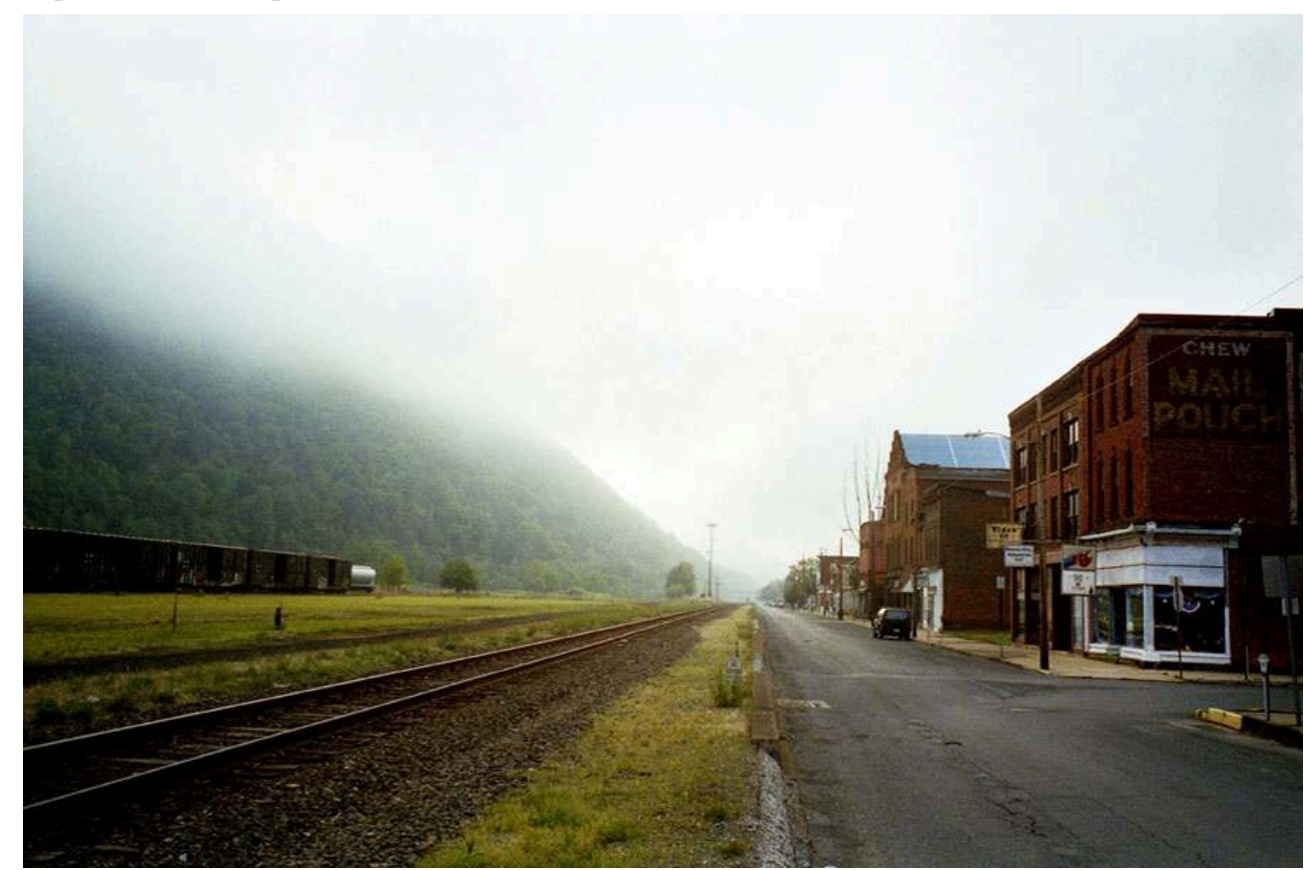


Richard Pak était allé aux Etats-Unis à reculons, en pensant n'avoir rien à y faire, et voilà qu'il y trouve toutes les représentations mentales issues de ses influences littéraires et cinématographiques. Il se prend au jeu de photographier, et le voici finalement à l'affût d'images familières qu'il reproduit avec délectation et insistance, sans volonté de subversion, juste avec l'envie de les retrouver (2003-2005 et 2007-2009). Il cherche là où le temps s'est arrêté, et entend fixer ces moments figés d'endroits sinistrés, le pittoresque sordide de personnages entrevus dans son imaginaire de lecteur ou de spectateur de films américains. Dans ses photographies, circule tout un imaginaire de l'Americana des années 50,60 ou 70, avec des poncifs assumés ${ }^{5}$ : un chemin de fer qui court vers l'ouest, parallèle à la désolation d'une main street unique, bordée de petits commerces aux devantures d'un autre âge, roadside diners isolés, trailerhomes délabrés-mobil-homes immobiles, comme dit Richard Pak-, serveuses seules et désabusées, ours empaillé, gueule grande ouverte, qui, loin d'être un trophée, est un voisin avec lequel il faut parfois composer. Même la technique de Richard Pak est d'antan puisqu'il a recours à l'argentique. Dans son travail, rien du mitraillage numérique : il choisit ses moments avec soin et parcimonie. Et n'a pas à les montrer à ses modèles.

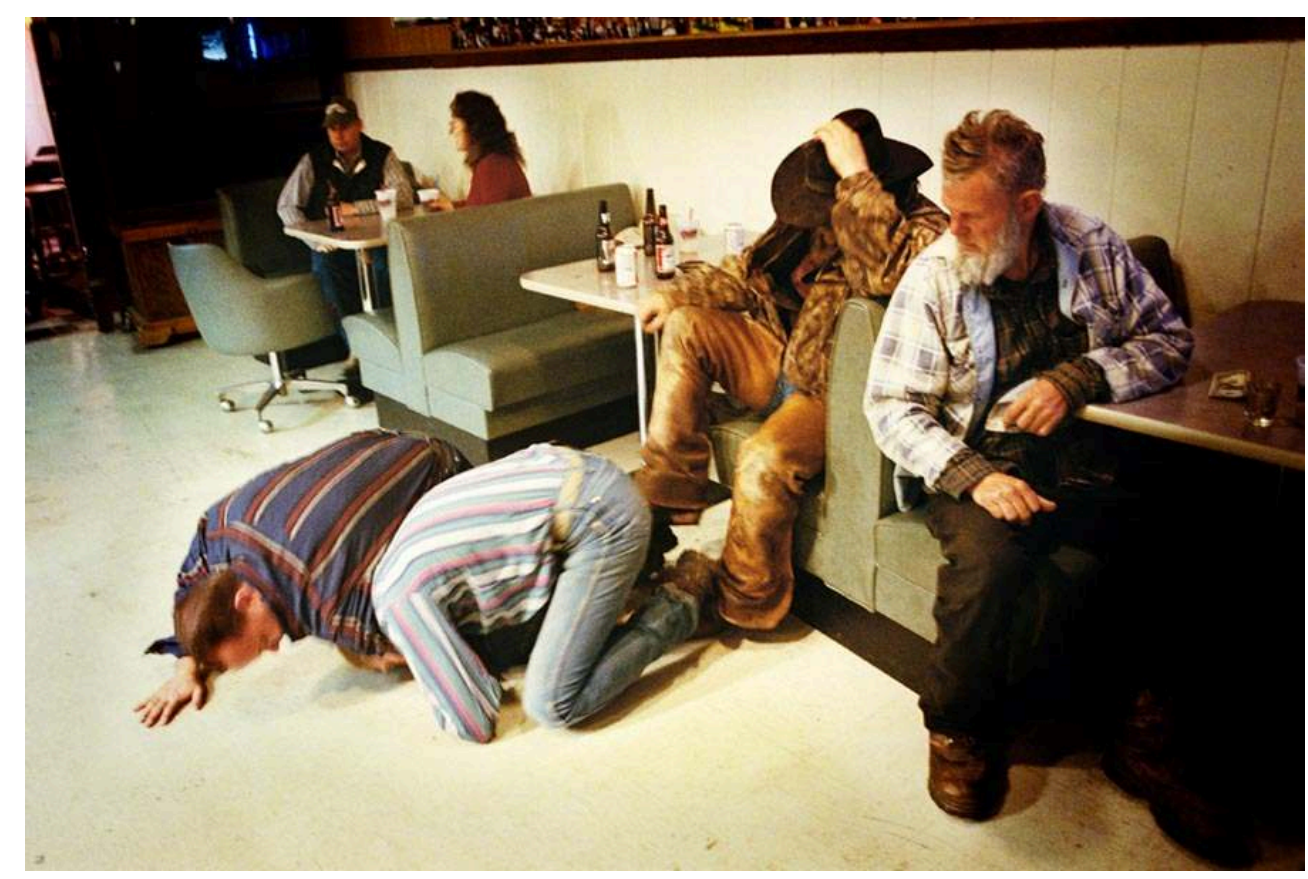

7 Ses photographies ne nous donnent pas vraiment accès aux personnes elles-mêmes. Plus que des personnes, elles nous donnent à voir un personnage, parfois une "gueule", un type. En témoignent l'importance du contexte et l'abondance des accessoires (tatouages, piercings...) qui composent les personnages autant que leur visage tendu. Dans ces décors sont retranscrits le banal, le quotidien, sans apologie aucune, mais avec tous les marqueurs caractéristiques des mondes dépeints. Par exemple, les mugs à cœur devant l'évier de cuisine propret, issus évidemment de la banlieue monotone de la middle-class aux pavillons sagement alignés, et aux pelouses bien arrangées, mais sans une once de fantaisie. Ou encore le rideau qui virevolte sous l'effet d'un courant d'air dans un trailer-home miteux au linoleum crasseux. Quelques images éparses mettent en perspective l'alignement des lattes de bois ou briques des murs des maisons comme autant d'illusoires lignes de fuite. Richard Pak ne dresse ni un état des lieux, ni un bilan. Il refuse tout étendard et revendique le caractère fictif de son travail. 


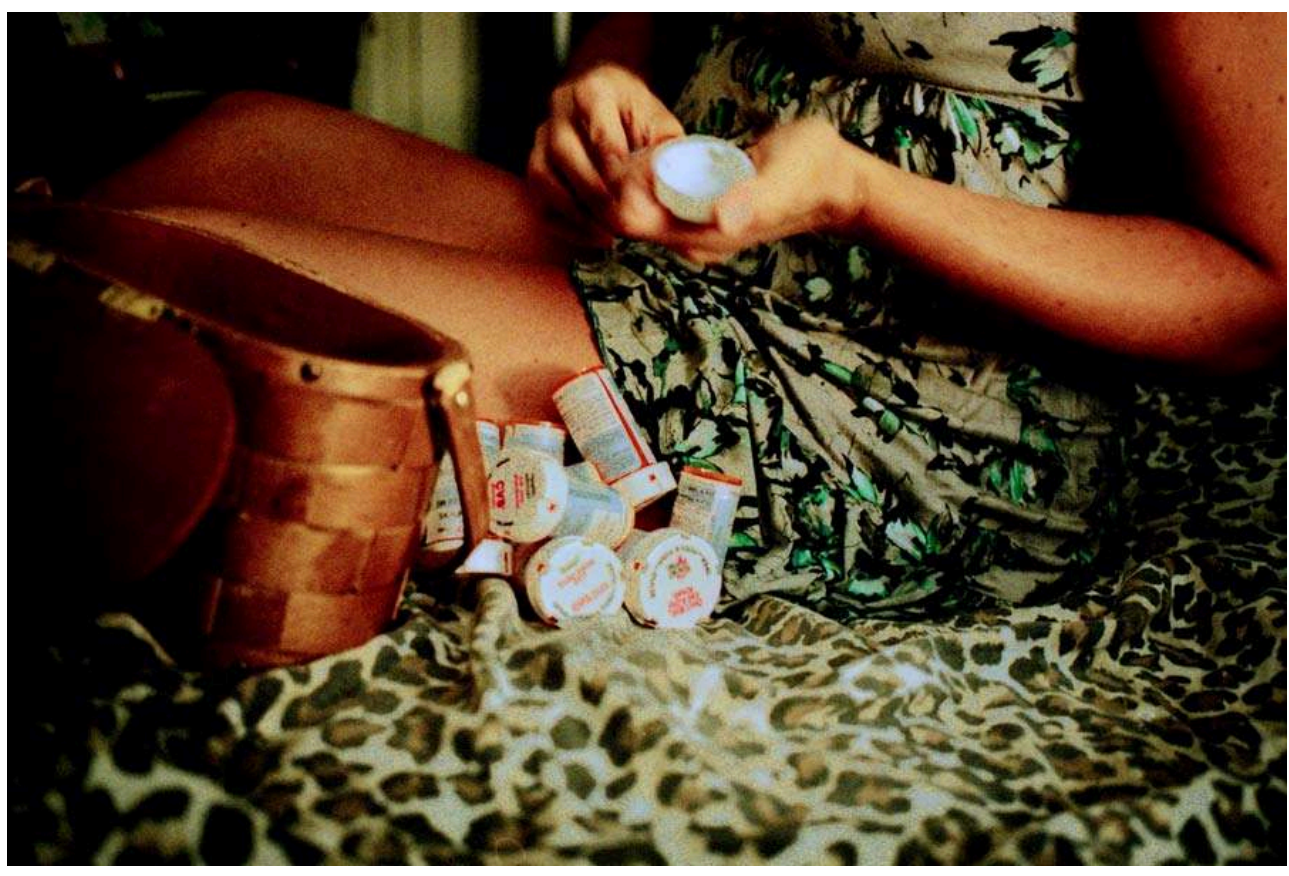

8 Il cherche ce qu'il connaît déjà mais il demande à le rencontrer en chair et en os, en bière, en joint et plus si affinités. Même s'il compte aussi sur la chance que les rencontres met sur sa route, Richard Pak a fait, pour son dernier voyage, des castings sur MySpace en amont de ses séjours, pour s'inviter dans le quotidien intime, claustrophobique et glauque de ces marginaux ou banlieusards qu'il photographie sans concession. En pénétrant dans leur vie, il réussit le tour de force de ne pas donner l'impression d'une intrusion. Sans doute car cette tranche de leur vie, il la partage, et partage aussi leur déchéance du moment, défonces et autres dérives. Il mêle scènes prises sur le vif en petit format et portraits frontaux en plan américain (pour employer la grammaire filmique) ou encore portraits en buste ou tronies en grands formats. Le spectre est relativement large-portraits de femmes et d'hommes, jeunes et moins jeunes, issus de la middle-class mais surtout de la frange sociale de la white trash un peu décalée: femme à l'œil au beurre noir, une nue au ventre vergéturé, une junkie rachitique, un transexuel après son opération, une femme aux seins augmentés artificiellement, une autre du quatrième âge alitée. Des personnages sans fards et miséreux, malmenés par le destin, englués dans leur condition, sans prise sur leur devenir, aux antipodes du self-made man et du life, liberty vantées aux côtés du pursuit of happiness. 


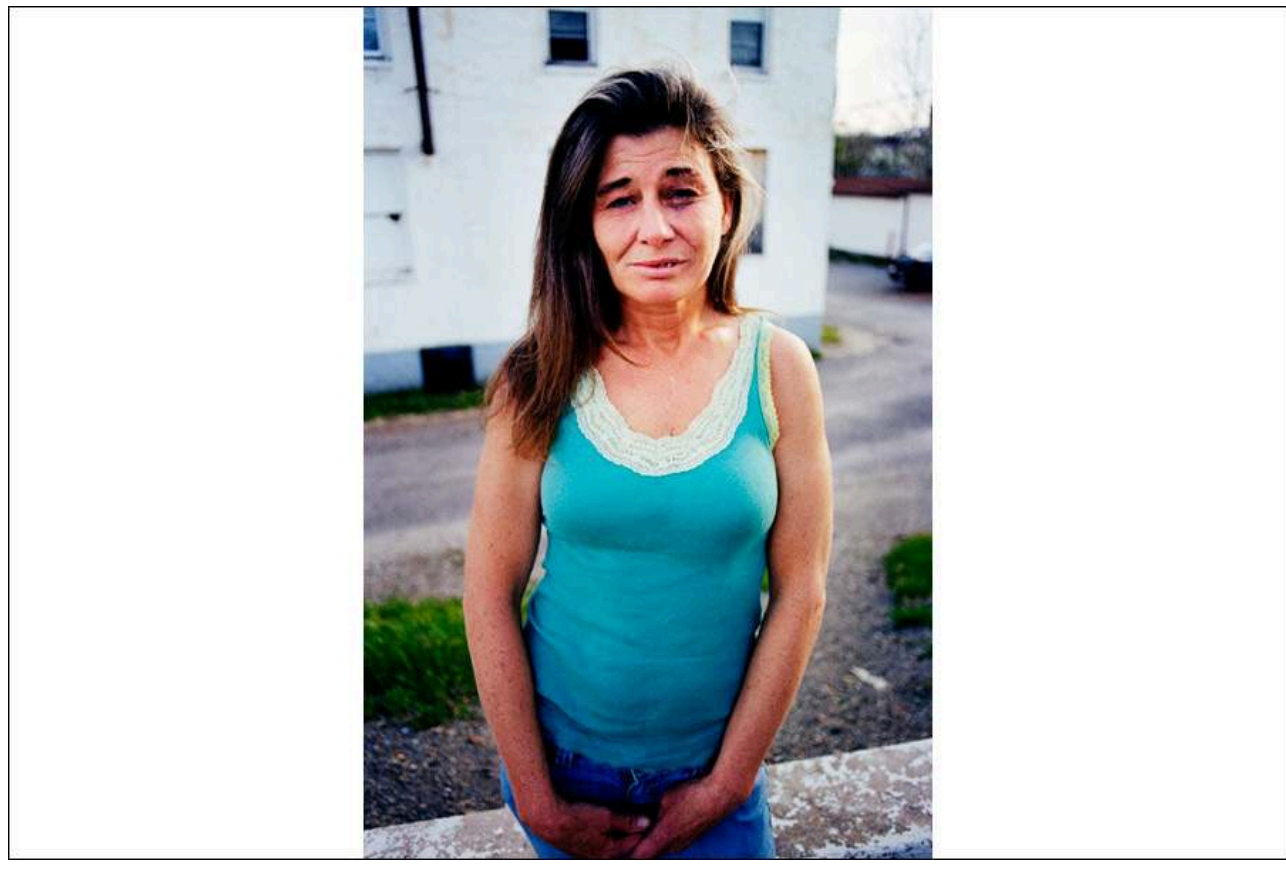

9 Des échantillons d'humanités, des portraits au réalisme cru, sans compromission. Le sujet est sordide, mais sa représentation n'est pas chagrine. D'où cela vient-il ? A part quelques photos au chromatisme vintage (par exemple, celle des deux jeunes gens dans la voiture rouge), les couleurs saturées (plus que n'en laissent présager les paroles de l'artiste qui dit préférer les tons pastels) retirent ce qu'il pourrait y avoir de blafard.

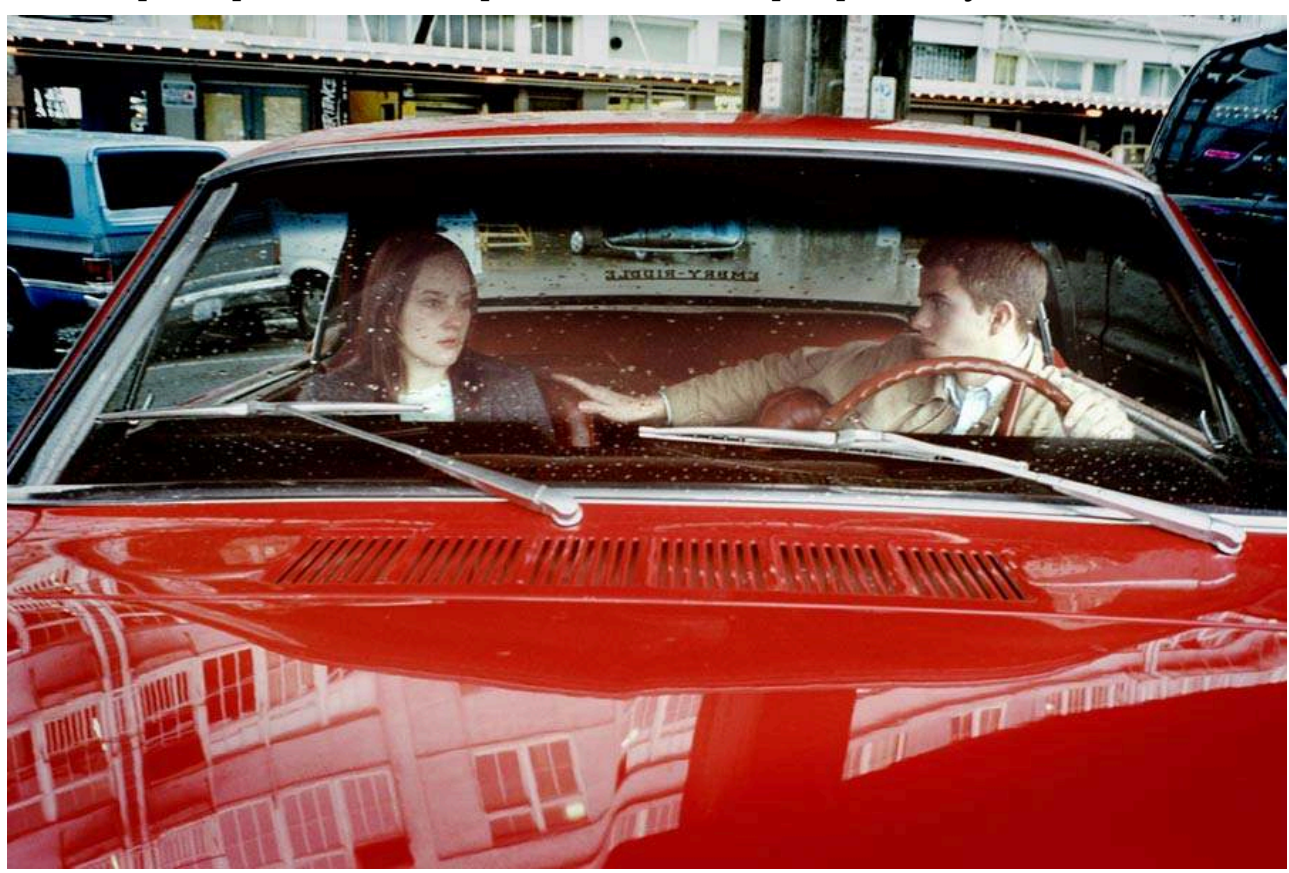

Mais c'est sans doute l'impression de déjà-vu traqué par l'objectif de Richard Pak, les associations à des univers cinématographiques ou romanesques familiers qui en dématérialisent le désarroi, le dénuement, la déréliction. Une femme allongée par terre, dont on ne sait pas si elle est sous emprise de l'alcool ou de stupéfiants, un enfant avec des marques de bronzage flottant face contre terre dans sa baignoire-tout cela relève plus des scènes droit sorties de films de Tarantino ou des frères Coen que de sinistres faits divers réels. 


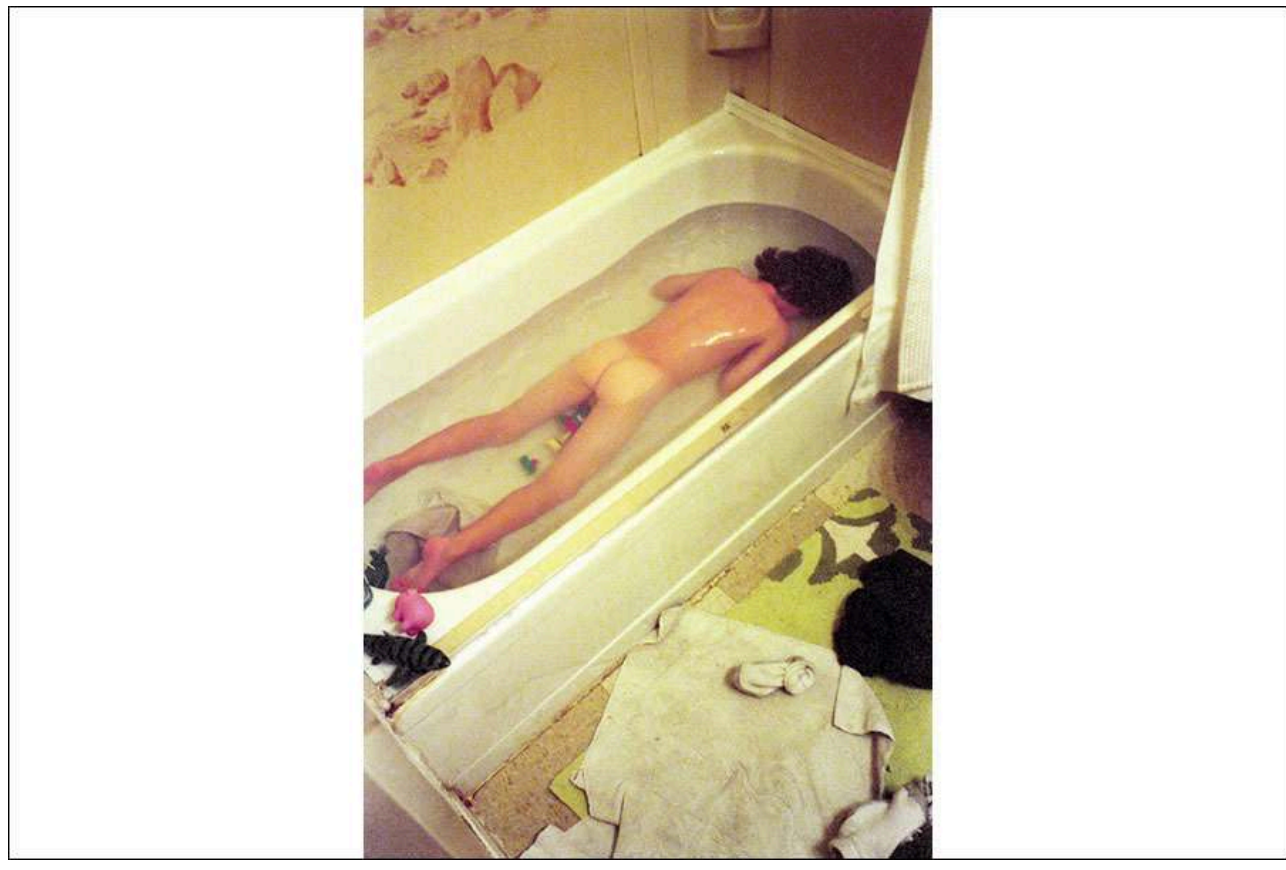

11 Cela est d'autant plus vrai que les images sont groupées en séquence, par trois, quatre ou cinq, sans cadre ou avec tout au plus un discret cadre américain. Et le visiteur est à l'image des deux personnages d'une des photographies, personnages sidérés, bouche bée devant leur écran télévisuel surexposé.

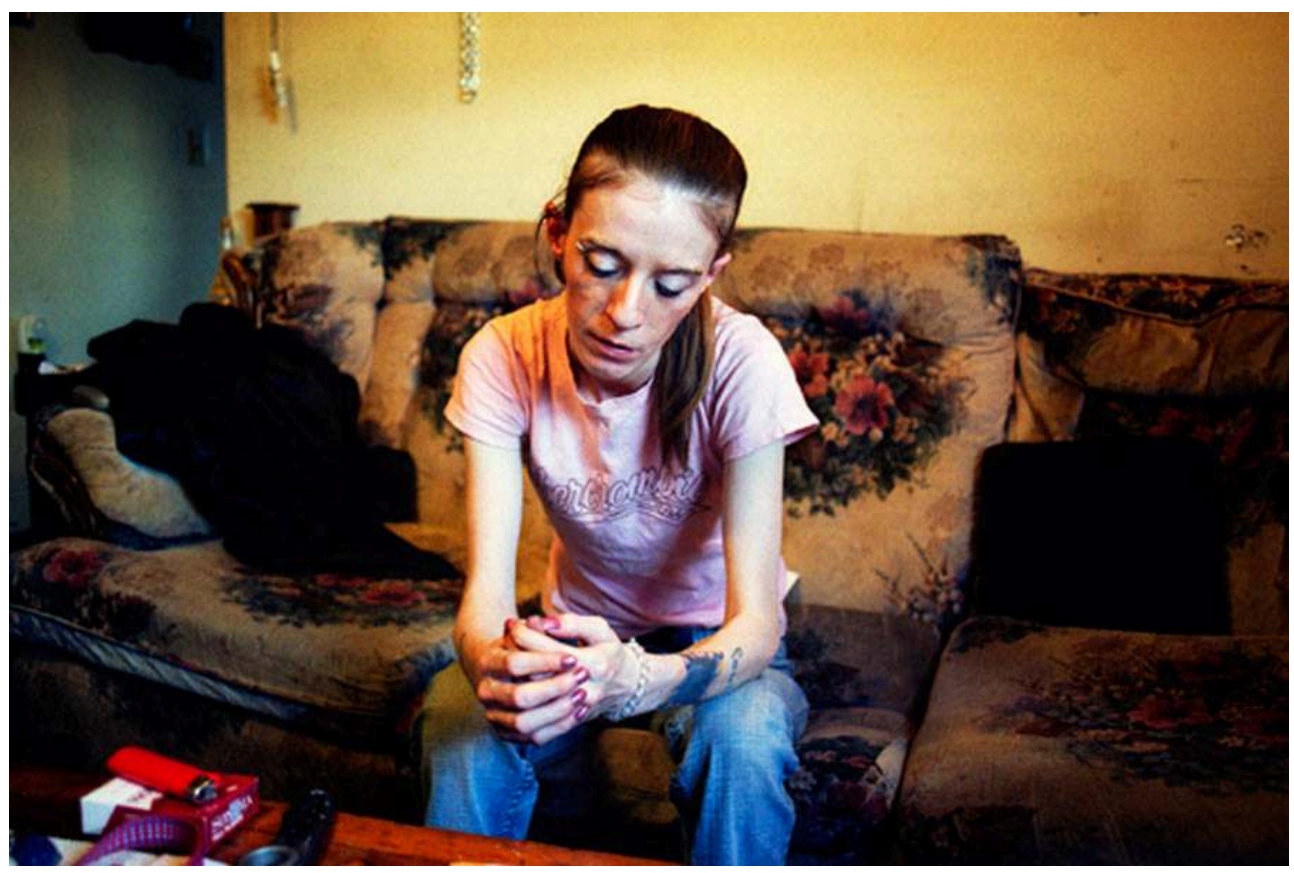

Please, Come back, le titre du catalogue, signale, certes, le désir de retenir un temps et un imaginaire passés, la volonté de le faire revenir par l'écriture et l'acte photographique, mais c'est aussi une phrase adressée par une de ses rencontres telle "un appel au secours", et dont le photographe nous dit qu'elle le mine encore. Elle résonne comme un aveu de la culpabilité qui hante tout photographe plutôt documentariste qui fait son miel du désarroi des autres - prédateur généreux et empathique, certes, mais chasseur d'images quand même. ${ }^{7}$ Il est venu, a vu, a fait ce qu'il avait à faire-traquer et arracher des images à des miséreux qui n'ont rien, et ce, sans contrepartie réelle, si ce n'est 
parfois l'illusion réconfortante d'un retour. Il n'y a aucune photo volée, dit-il. Et il est vrai qu'il partage, mais des bières, des joints, voire des drogues plus nocives-autant de produits qui ne sont que de destructeurs échappatoires. Quelle générosité $\mathrm{y}$-a $\mathrm{t}$-il dans le partage éphémère d'une déchéance ? Le photographe est témoin social, mais tous ces délaissés, il les a abandonnés, lui aussi, à leur piteux sort. Richard Pak en est conscient, et il ne reviendra pas sur les lieux, dit-il dans un passage de son journal ${ }^{8}$. Ils resteront les figurants piteux, pouilleux, indigents qu'ils sont. Le passage du photographe ne leur aura rien fait gagner.

Une serveuse d'un coffee shop miteux "aux airs de Gena Rowlands" (62) raconte que sa mère a été photographiée elle aussi autrefois; elle ne connaît pas le nom du photographe, mais le rapporte à Richard Pak le lendemain, après avoir demandé autour d'elle. "C'était un certain Avedon (...) Ça te dit quelque chose ?"

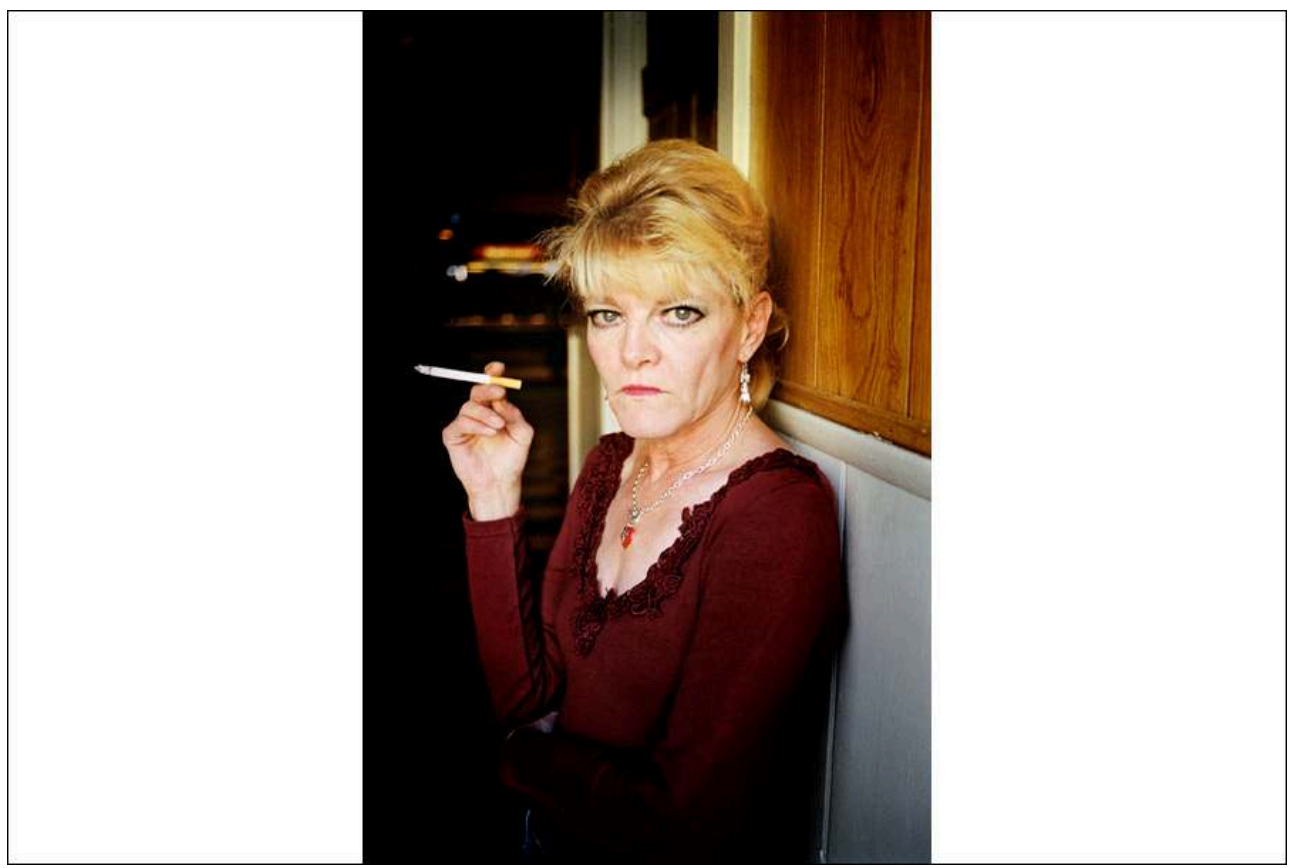

Pour finir, quelques images du catalogue jurent sur le reste : une image crue de couple nu à la manière de Nan Goldin, un flou de mer en noir et blanc au flux poétique à la Bernard Plossu, dont on comprend mal la présence dans cette série ; elles en rompent l'homogénéité, sans que l'on voie ce que cela apporte. Le directeur et le photographe ont eu la bonne idée de ne pas les faire figurer dans l'exposition. Un autre mérite de l'exposition est d'être la première à présenter le texte, dont Richard Pak dit qu'il est devenu indissociable de son œuvre photographique.

Cette exposition correspond bien à la prédilection actuelle du Château d'eau pour les photographies à la lisière de la fiction et du documentaire. Elle est couplée, comme toujours en ce lieu, à une autre exposition d'un artiste jeune et moins connu, dans le cas présent, à Gaël Bonnefon. La deuxième exposition fait parfois office de faire-valoir. Ici, ce n'est pas le cas, puisque Gaël Bonnefon surfe sur un créneau similaire et même verse davantage encore dans le sordide avec des couleurs parfois criardes. Ce voisinage finalement les dessert un peu tous les deux.

L'exposition Pursuit a déjà été présentée à Brest, Lorient, Paris, en Belgique et en Lituanie. 


\section{Références du catalogue}

\section{NOTES}

1. Sur une autre photo, HOPE est également écrit en petites lettres sur le mur d'une femme du 4ème âge alitée.

2. Déclaration d'indépendance des Etats-Unis, 4 juillet 1776 : "We hold these truths to be selfevident, that all men are created equal, that they are endowed by their Creator with certain unalienable Rights, that among these are Life, Liberty, and the pursuit of Happiness."

3. "Il n'a jamais entendu parler de Raymond Carver ni de John Fante" (66) "Je l'écoute, silencieux, attentif et impuissant, héros malgré moi d'une nouvelle de Carver (...)” (67) Interrogé sur ses influences, Richard Pak répond: "J'ai aimé les portraits d'Eggleston autrefois, mais mes photographes préférés, c'est Carver, c'est Faulkner, c'est Altman etc.." Question posée par l'auteur au photographe le 29 novembre 2012.

4. "La serveuse aux airs de Gena Rowlands" (62). "[...] donne à la scène un aspect assurément cinématographique" (66). "Conscient de la force de cette scène comme sortie d'un film des 70s je les photographie frénétiquement (...)" (76).

5. “je dîne dans un roadside diner des années cinquante parfaitement préservés" (61); "j'ai élu domicile au Custer Saloon, un vieux bar où le temps semble s'être arrêté boire dans les années 70 et n'en est jamais reparti."

6. "Dans un murmure Della me glisse à l'oreille 'please, come again' comme un appel au secours qui me sert encore la gorge." (72).

7. Voir la dimension métapicturale de l'ours empaillé, ou des punaises plantées en masse dans un poteau.

8. "Je prends conscience que ce voyage est le dernier de ce projet" (61). 
9. "Elle m'annonce, sur le ton de la confidence, qu'elle a appelé sa mère pour connaître le nom du type qui l'avait photographiée. C'est un certain Richard Avedon et le bouquin The American West ou un truc du genre. Ça te dit quelque chose?"' (63).

\section{INDEX}

Mots-clés : cinéma New Hollywood, John Fante, photographie, Raymond Carver, Richard Pak, Truman Capote

Keywords : John Fante, New Hollywood film, photography, Raymond Carver, Richard Pak, Truman Capote

Thèmes : Trans'Arts

\section{AUTEUR}

MURIEL ADRIEN

Université de Toulouse 2-Le Mirail 